\title{
VARIAÇÃO DA SOBRECARGA DE TREINAMENTO NO COM- PORTAMENTO DA FORÇA MUSCULAR E DA PERCEPÇÃO SUBJETIVA DE DOR EM MULHERES SEDENTÁRIAS
}

\author{
VARIATION OF THE TRAINING OVERLOAD IN THE BEHAVIOR OF THE MUSCULAR FORCE \\ AND SUBJECTIVE PERCEPTION OF PAIN IN SEDENTARY FEMALE
}

Alex Souto Maior ${ }^{1}$, Thiago Menuci², Vinícius Soares², Lucas Rafael de Souza², Márcio Gribov², Roberto Simão ${ }^{3}$

\begin{abstract}
1Doutorando em Ciências Biológicas (Fisiologia) do Instituto de Biofísica Carlos Chagas Filho da Universidade Federal do Rio de Janeiro (IBCCF/UFRJ). ${ }^{2}$ Graduados em Educação Física e Especialistas em Treinamento de Força da Universidade Gama Filho. ${ }^{3}$ Doutor e Professor Adjunto da Escola de Educação Física e Desportes da Universidade Federal do Rio de Janeiro (EEFD/UFRJ).

CorRespondÊncia: Alex Souto Maior. Av. Marechal Djalma Ribeiro, $n^{\circ} 25$, apto 103. Recreio dos Bandeirantes, Rio de Janeiro/RJ. Cep: 22790-790. alex.bioengenharia@terra.com.br
\end{abstract}

Maior AS, Menuci T, Soares V, Souza LR, Gribov M, Simão R. Variação da sobrecarga de treinamento no comportamento da força muscular e da percepção subjetiva de dor em mulheres sedentárias. Medicina (Ribeirão Preto) 2008; 41 (2): 168-76.

RESUMO: O objetivo do estudo foi analisar e comparar os ganhos de força e observar o comportamento da percepção subjetiva de dor (PSD) no período de oito semanas em mulheres sedentárias através de dois programas distintos de exercícios resistidos. O estudo foi composto de 16 mulheres $\left(27,5 \pm 4,8\right.$ anos; $\left.57 \pm 3,9 \mathrm{~kg} ; 162,8 \pm 3,3 \mathrm{~cm} ; 21,4 \pm 1, \mathrm{~kg} / \mathrm{m}^{2}\right)$, aparentemente saudáveis e sedentárias. Elas foram separadas aleatoriamente em dois grupos distintos: Grupo 1 (G1) - os indivíduos nas duas primeiras semanas realizaram duas séries em 10 repetições com carga que variava de 40 a $60 \%$ do peso corporal. Entre a $3^{\mathrm{a}}$ e a $4^{\mathrm{a}}$ semana treinaram com três séries de 10 repetições a $80 \%$ de $1 \mathrm{RM}$ (repetição máxima). Entre a $5^{\mathrm{a}}$ e a $8^{\mathrm{a}}$ semana ocorreu aumento progressivo do número de séries para quatro, mantendo o número de repetições (10 repetições) e a mesma sobrecarga; Grupo 2 (G2) - foi realizado um programa de treinamento contínuo de três séries de 10 repetições nas duas primeiras semanas com uma carga que variava de 40 a $60 \%$ do peso corporal. Nas semanas subseqüentes o treinamento foi alterado para quatro séries de 10 repetições com uma carga equivalente a $80 \%$ de $1 \mathrm{RM}$ ao longo das oito semanas de treinamento. Para análise da força muscular foi utilizado o teste de 1RM no final da $2^{\mathrm{a}}, 4^{\mathrm{a}}, 6^{\mathrm{a}}$ e $8^{\mathrm{a}}$ semanas com intuito de verificar o comportamento da força muscular e otimizar a carga de treinamento. Os exercícios utilizados no protocolo de treinamento e no teste de 1RM foram: leg press $45^{\circ}$, extensão de joelho, flexão de joelho, puxada pela frente, flexão de cotovelo e extensão de cotovelo. A PSD foi apresentada a cada voluntário $48 \mathrm{~h}$ pós a realização de cada teste de 1RM. A ANOVA Two Way seguida do teste post hoc de Bonferroni verificou que os resultados intra-grupos para G1 apresentaram diferença significativa para todos os exercícios em todos os testes. Contudo, em G2 a diferença significativa foi observada, em grande parte dos exercícios, somente do $3^{\circ}$ e $4^{\circ}$ teste em relação ao $1^{\circ}$ teste. Para as análises inter-grupos o teste Mann-Whitney para amostras independentes verificou ganhos de força significativo do G1 em relação a $\mathrm{G} 2$ nos exercícios leg press $45^{\circ}$, rosca tríceps e rosca bíceps. Em relação a PSD foi observada uma queda significativa na escala analógica de $\mathrm{G} 1$ em relação a $G 2(p<0,001)$. Em conclusão, a sobrecarga progressiva imposta ao $\mathrm{G} 1$ promoveu significativos ganhos de força muscular e menor PSD em relação ao $\mathrm{G} 2$.

Descritores: Força Muscular. Exercício Físico. Dor Muscular Tardia. 


\section{1- INTRODUÇÃO}

Os exercícios resistidos (ER) têm demonstrado ser benéficos na melhoria de vários parâmetros funcionais, bem como o aumento da massa muscu$\operatorname{lar}^{1,2}$. Contudo, outros estudos demonstram papel relevante no sistema neuromuscular ${ }^{3}$, cardiovascular $^{4} \mathrm{e}$ metabólico ${ }^{5}$.

Os ER são utilizados como um meio efetivo de incremento da força muscular e melhoria do estado funcional em todas as faixas etárias. Isto justifica a necessidade da utilização de sobrecargas na prescrição do treinamento, com objetivo de melhorar o desempenho físico associado ao aumento da força e potência muscular ${ }^{3,6}$. No entanto, ER se referem a uma modalidade de atividade física sistematizada composta de variáveis (volume, intensidade, freqüência, duração, recuperação, ordem dos exercícios, equipamentos e tipo de treinamento) que precisam ser bem controladas, para que possam produzir efeitos benéficos ${ }^{7}$. Assim, alguns posicionamentos como do American College of Sports Medicine (ACSM) ${ }^{1}$ e o estudo de Kemmler et al. ${ }^{8}$ evidenciam que o total de carga utilizada para um exercício específico é, provavelmente, a variável mais importante.

Durante os ER, para que ocorra resposta aos estímulos, os músculos respondem através da atividade neural, ou seja, ocorre uma integração entre os sistemas sensoriais e o cortéx motor com uma resposta de controle antecipatória ${ }^{2,9,10}$. A mecanismo de adaptação do sistema nervoso apresenta predominio significativo durante as fases iniciais do treinamento de força, em que sua efetiva intervenção auxília no aumento da força muscular sem mudança significativa na área de secção transversa do músculo ${ }^{9,10}$. As fases intermediárias e avançadas dos ER (após adaptação neural) passam a ser prioridade dos fatores hipertróficos ${ }^{2,10}$, assim, reduzindo a freqüência do estímulo neural, em relação ao início do treinamento.

Muitos estudos verificaram a importância da adaptação dos mecanismos neurais relacionado aos ganhos de força muscular e as semanas iniciais ao treinamento ${ }^{11,12,13}$. Desta forma, estas conclusões relatam uma melhora relevante no padrão funcional e do desempenho físico. Assim, Gruber et al. ${ }^{12}$ observaram que indivíduos após quatro semanas de treinamento envolvendo os ER apresentaram significativo aumento da força muscular. O estudo de Loveless et al. ${ }^{13}$ corroborou com o estudo supracitado, em relação ao aumento de força muscular. Contudo neste estudo o tempo de treinamento foi de oito semanas. De acordo com estes estudos citados o aumento na força muscular é proporcional ao potencial evocado pelo sistema nervoso central, a sincronização das unidades motoras, à quantidade de sobrecarga, o comportamento da força absoluta e relativa desenvolvida e o número de contrações musculares executadas durante o ER 2,3,7,11-13. Assim, o ganho de força muscular associado à resposta neural apresenta alto índice de correlação entre quatro e oito semanas ${ }^{9-13}$.

Contudo, a progressão da sobrecarga utilizada na prescrição do treinamento em ER proporciona economia no potencial evocado pelo sistema nervoso central, consequentemente, menor dano muscular, menor dispêndido energético e maior recrutamento de unidades motoras ${ }^{14}$. Estes fatores proporcionam adaptações em níveis de coordenação motora (inter e intramuscular) e melhor eficiência de estimulação das unidades motoras ${ }^{2,12,14}$.

Outro fator de grande relevância na relação do comportamento de força muscular e ER é o nível de dor muscular. Ou seja, é relatado em estudos que grupos musculares submetidos a intensa sobrecarga apresentam acometimentos de dor muscular ${ }^{15,16}$. Estes grupamentos musculares apresentam sensibilidade a toque e redução da amplitude articular com apresenta capacidade reduzida de promover força muscular ${ }^{16}$.

Em suma o objetivo do estudo foi analisar e comparar os ganhos de força e observar o comportamento da percepção subjetiva de dor (PSD) no período de oito semanas em mulheres sedentárias através da sobrecarga imposta na execução dos exercícios resistidos em dois programas de treinamento distintos. Desta forma, determinar o protocolo de treinamento que apresenta melhor desempenho em relação à força muscular com menor potencial de proporcionar dor muscular tardia.

\section{2- MATERIAIS E MÉTODOS}

\section{1- Sujeitos}

O estudo foi composto de 16 mulheres $(27,5 \pm$ 4,8 anos; $\left.57 \pm 3,9 \mathrm{~kg} ; 162,8 \pm 3,3 \mathrm{~cm} ; 21,4 \pm 1, \mathrm{~kg} / \mathrm{m}^{2}\right)$, aparentemente saudáveis e sedentárias. Para análise das variáveis antropométricas foram utilizados um estadiômetro graduado em milímetros (verificação da estatura) e uma balança digital (Filizola). Para melhor homogeneidade da amostra foi adotado como critério de exclusão:

1- portadoras de doenças degenerativas; 2- portadoras de lesões osteomioarticulares; 
3- submissão a cirurgias articulares a menos de 12 meses;

4- portadoras de disfunção cardiovascular.

Os indivíduos foram esclarecidos sobre todo protocolo de treinamento e responderam negativamente o questionário PAR-Q. Contudo, assinaram o termo de consentimento para pesquisas com seres humanos relativo ao Ministério da Saúde na resolução $\mathrm{n}^{\circ} 196 / 96$.

\section{2- Teste de 1RM (repetição máxima) e Per- cepção subjetiva de dor}

Foi utilizado como método não invasivo de análise de força muscular o teste de 1RM. O procedimento do teste consistia de três a quatro tentativas, em que foi validado como carga máxima o deslocamento de carga obtido na última execução a partir da falha concêntrica. A carga inicial (aquecimento articular) para o teste consistia de $30 \%$ da massa corporal de modo que o individuo conseguia realizar o movimento confortavelmente, assim, ocorreu uma progressão de carga de forma que o indivíduo realizasse 1RM. A cada nova tentativa realizava-se adição de incrementos progressivos de $5 \mathrm{~kg}$, sendo dado um intervalo de 3 a 5 minutos entre cada tentativa ${ }^{3}$.

Com o objetivo de reduzir a margem de erro no teste de 1RM adotaram-se as seguintes estratégias:

1- Instruções padronizadas foram oferecidas antes dos testes, de modo que o avaliado estivesse ciente de toda a rotina que envolvia a coleta de dados;

2- $\mathrm{O}$ avaliado foi instruído sobre a técnica de execução do exercício, inclusive realizando-o algumas vezes sem carga, para reduzir um possível efeito do aprendizado nos escores obtidos;

3- $\mathrm{O}$ avaliador estava atento quanto à posição adotada pelo praticante no momento da medida. Desta forma, pequenas variações no posicionamento das articulações envolvidas no movimento poderiam acionar outros músculos e levar a interpretações errôneas de escores obtidos;

4- Para estabelecer a carga que gerava a força máxima no teste de 1RM utilizou-se os equipamentos da marca Word Fitness (Brasil).

O teste de 1RM foi aplicado nos seguintes exercícios: leg press $45^{\circ}$, extensão de joelho (cadeira extensora), flexão de joelho (cadeira flexora), puxada pela frente, flexão de cotovelo (rosca bíceps), extensão de cotovelo (rosca tríceps). Para tanto os exercícios serão descritos relacionando a posição inicial (PI) e fase concêntrica (FC).

\section{Leg press $4^{\circ}$ :}

a) PI - O indivíduo sentado no banco em um ângulo de $45^{\circ}$, pernas paralelas, com um pequeno afastamento lateral e com os joelhos flexionados $\left(80^{\circ}\right.$ entre a perna e coxa), braços ao longo do corpo segurando a barra de apoio; b) FC -A partir da PI $\left(80^{\circ}\right.$ entre a perna e coxa), realizava-se a extensão completa dos joelhos e quadris.

\section{Extensão de joelho:}

a) PI - os indivíduos sentados no equipamento com braços ao longo do corpo, segurando o apoio do aparelho; tronco com inclinação de $70^{\circ}$ e joelhos flexionados em $90^{\circ}$, com a cabeça posicionada no plano de Frankfurt; b) FC - a partir da posição inicial, realizava-se a extensão completa dos joelhos.

\section{Flexão de joelho:}

a) PI - os indivíduos adotaram a posição de decúbito ventral no equipamento com braços flexionados segurando o apoio do aparelho, os joelhos mantiveram-se em extensão; b) FC - a partir da posição inicial, realizava-se a flexão completa dos joelhos.

\section{Puxada pela frente:}

a) PI - Sentado no aparelho com os braços elevados e cotovelos estendidos com as mãos pronadas segurando na barra longa; b) FC - A partir da posição inicial realizava-se a adução das escapulas, com flexão dos cotovelos até a região do manúbrio. Contudo, qualquer variação do deslocamento do tronco durante a realização do teste proporcionava a realização de um novo teste.

\section{Rosca bíceps (flexão de cotovelos):}

a) PI-O indivíduo em pé, pernas paralelas com um pequeno afastamento lateral, com os joelhos estendidos, quadris na posição anatômica, braços ao longo do corpo com as mãos supinadas segurando a barra e a cabeça posicionada com o plano de Frankfurt; b) FC - A partir da posição inicial, realizava-se a flexão completa dos cotovelos.

\section{Rosca tríceps (extensão de cotovelos):}

a) PI - O indivíduo em pé, pernas paralelas com um pequeno afastamento lateral, com os joelhos estendidos, quadris na posição anatômica, braços flexionados a $90^{\circ}$ segurando a barra com as mãos na posição supina e a cabeça posicionada com o plano de Frankfurt; b) FC - A partir da posição inicial, realizava-se a extensão completa dos cotovelos.

Como proposto por Cook et al ${ }^{15}$ foi utilizada, no pós-treinamento, a escala analógica de percepção subjetiva de dor (PSD) que apresenta uma variação 
de 0 (sem comprometimento de dor) a 10 (dor extremamente intensa). Esta escala foi apresenta $48 \mathrm{~h}$ após cada sessão de teste de 1RM (total de 4 testes). Assim, para melhor apresentação dos resultados cada dia que a escala de PSD foi argüida pelo voluntário foi utilizada a nomenclatura $1^{\circ}$ dia, $2^{\circ}$ dia, $3^{\circ}$ dia e $4^{\circ}$ dia, respectivamente.

\section{3- Protocolo de treinamento}

Os indivíduos realizaram o programa de exercícios de força durante o período de oito semanas (3 vezes por semana) e foram divididos em dois grupos aleatórios:

1- Grupo $1(\mathrm{G} 1: \mathrm{n}=08)$ : Foi realizado um treinamento respeitando o princípio da sobrecarga (aumento progressivo do volume de treinamento), ou seja, nas duas primeiras semanas os indivíduos realizaram duas séries de 10 repetições com uma carga que variava de 40 a $60 \%$ do peso corporal. $\mathrm{Na} 3^{\mathrm{a}}$ e $4^{\mathrm{a}}$ semana treinaram com três séries de 10 repetições a $80 \%$ de $1 \mathrm{RM}$. Da $5^{\mathrm{a}}$ a $8^{\mathrm{a}}$ semana ocorreu aumento progressivo do número de séries para quatro e foram mantidos o número de repetições $(10$ repetições) com o mesmo valor de sobrecarga ( $80 \%$ de 1RM).

2- Grupo 2 (G2: $n=08)$ : Foi realizado um programa de treinamento contínuo de 3 séries de 10 repetições nas duas primeiras semanas com uma carga que variava de 40 a $60 \%$ do peso corporal. Nas semanas subseqüentes o treinamento foi alterado para 4 séries de 10 repetições com uma carga equivalente a $80 \%$ de $1 \mathrm{RM}$ ao longo das oito semanas de treinamento.

Em ambos os grupos foram adotados os intervalos de dois minutos entre as séries e exercícios do protocolo dos ER. O teste de 1RM foi aplicado ao final da segunda, quarta, sexta e oitava semanas com intuito de verificar o comportamento da força muscular a partir do deslocamento de cargas e otimizar a carga de treinamento.

\section{4- Análise Estatística}

Os dados foram expressos em média e desvio padrão (descrição da amostra). Contudo, para análises intra-grupos foi adotado um ANOVA Two Way seguida do teste post hoc de Bonferroni. Para a relação comparativa inter-grupos (G1 Vs. G2) no teste de 1RM e na PSD foi utlizado o test MannWhitney para amostras independentes. A análise do $\Delta \%$ foi realizada através do cálculo: (momento $2-$ momento 1 / momento $1 \times 100)$. Para toda análise foi adota um nível de significância de $5 \%(\alpha=0,05)$ e o software Graph Pad Prisma 4.

\section{3- RESULTADOS}

\section{1- Análise intra-grupos e inter-grupos - teste de 1RM}

Os resultados intra-grupos para G1 apresentaram diferença significativa para todos os exercícios (membros inferiores e superiores) em todos os testes (Figura 1 e 2). Assim, a verificação do quarto teste em relação ao primeiro teste demonstrou aumentos percentuais significativos para: leg press $45^{\circ}(53,3 \%)$, flexão de joelhos (52,2\%), extensão de joelhos $(30,8 \%)$, puxada pela frente $(44,9 \%)$, rosca bíceps $(60,9 \%)$ e rosca tríceps $(49,8 \%)$ (Figura 1 e 2). Contudo, os resultados intra-grupos para G2 apresentaram, em grande parte dos exercícios, diferença significativa apenas em relação ao $1^{\circ}$ e $2^{\circ}$ teste, consequentemente, apresentando um nível de aparente estabilidade da força muscular em relação ao teste de 1RM (Figura 1 e 2). Apenas o exercício de rosca bíceps quando comparado o quarto teste em relação ao primeiro, segundo e terceiro teste apresentou aumento significativo em relação a progressão do treinamento $\left(\Delta \% 4^{\circ}\right.$ teste Vs. $1^{\circ}$ teste $\left.=24,1 \%\right)($ Figura 2$)$.

As análises inter-grupos foram observados aumentos significativos da força muscular no teste de 1RM para o exercício de Leg press $45^{\circ}$ no G1 em relação ao $\mathrm{G} 2(25,2 \%$ e $28,3 \%$, respectivamente) no $3^{\circ}$ e $4^{\circ}$ teste. Contudo, para o exercício de rosca tríceps este aumento foi significativo no $2^{\circ}$ teste $(42,9 \%), 3^{\circ}$ teste $(52,6 \%)$ e $4^{\circ}$ teste $(66,3 \%)$ (Tabela I). No exercício de rosca bíceps o aumento significativo foi observado somente no $4^{\circ}$ teste $(27,8 \%)$ (Tabela I).

\section{2- Análise intra-grupos e inter-grupos - PSD}

Os resultados intra-grupos para G1 apresentaram diferença significativa $(\mathrm{p}<0,0001)$ revelaram uma queda significativa na PSD do $1^{\circ}$ dia em relação ao $2^{\circ}$ dia $(44,2 \%), 3^{\circ}$ dia $(57,1 \%)$ e $4^{\circ}$ dia $(76 \%)$ (Figura 3$)$. Contudo, no G2 ocorreu diferença significativa $(p<0,0001)$ apenas do $1^{\circ}$ dia em relação ao $4^{\circ}$ dia $(31,4 \%)$. As análises inter-grupos (G1 Vs. G2) revelaram diferença significativa no $2^{\circ}$ dia $(45,9 \%), 3^{\circ}$ dia $(46,4 \%)$ e $4^{\circ}$ dia (40\%) (Figura 3). 


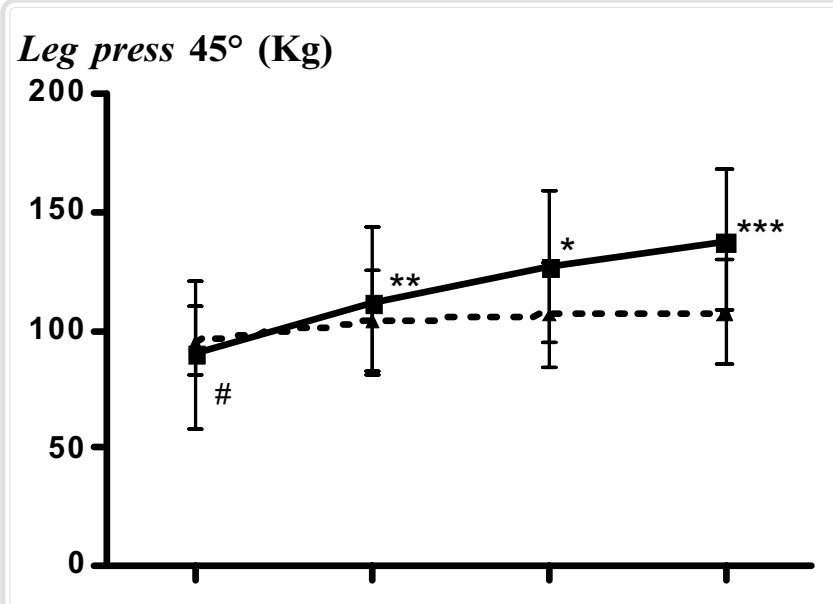

Flexão de joelho $(\mathrm{Kg})$

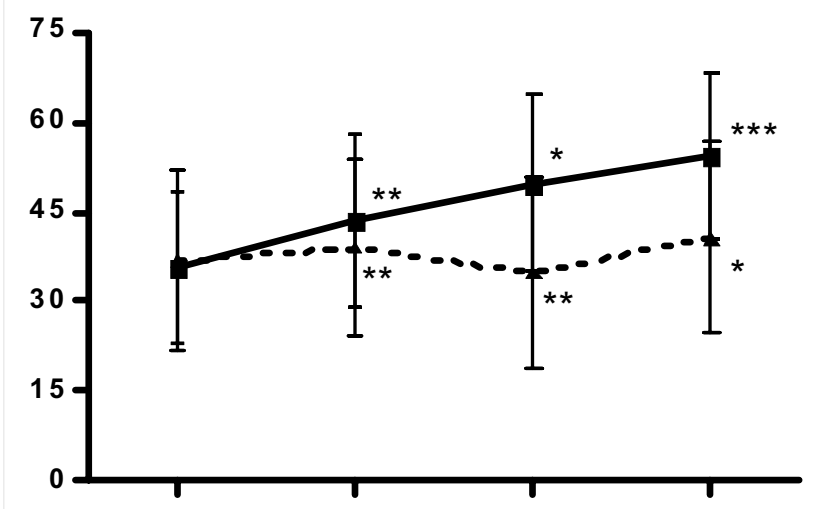

Extensão de joelho (Kg)

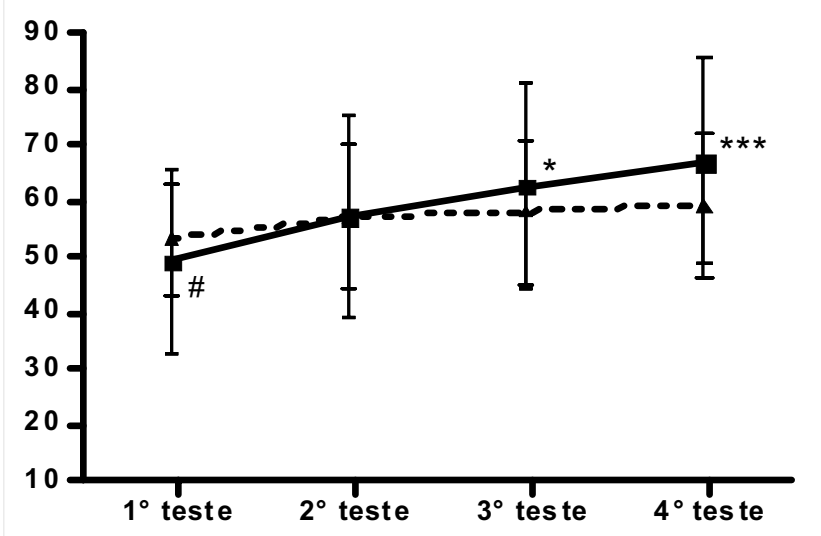

Figura 1: Análise intra-grupos com descrição por média e desviopadrão para os exercícios de leg press $45^{\circ}$, flexão de joelho e extensão de joelhos. Linha cheia - G1 $(n=08)$; Linha tracejada $\mathrm{G} 2$ ( $\mathrm{n}=08)$.

\# diferença significativa em relação aos demais testes $(p<0,05)$.

* diferença significativa em relação ao $1^{\circ}$ e $2^{\circ}$ teste $(p<0,05)$.

** diferença significativa em relação ao $1^{\circ}$ teste $(p<0,05)$.

${ }^{* * *}$ diferença significativa em relação ao $1^{\circ}, 2^{\circ}$ e $3^{\circ}$ teste $(p<0,05)$.

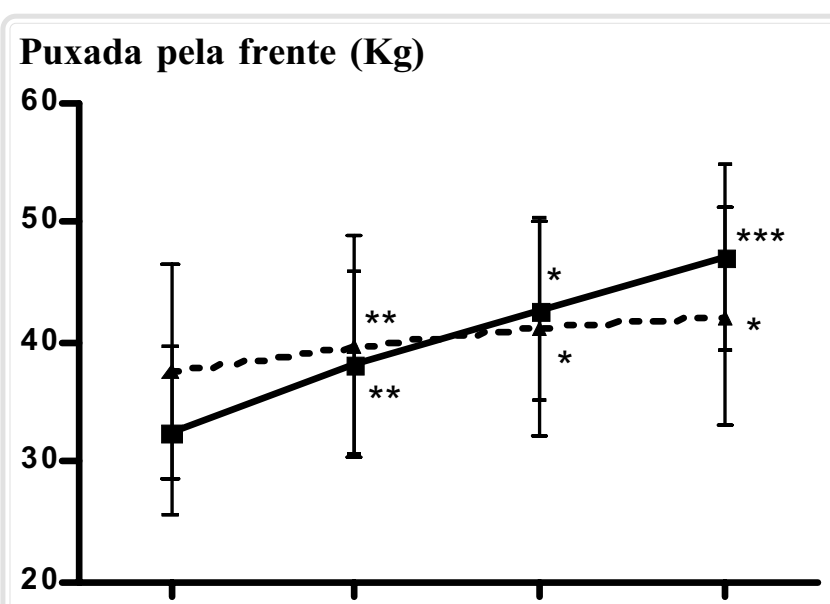

Rosca bíceps (Kg)

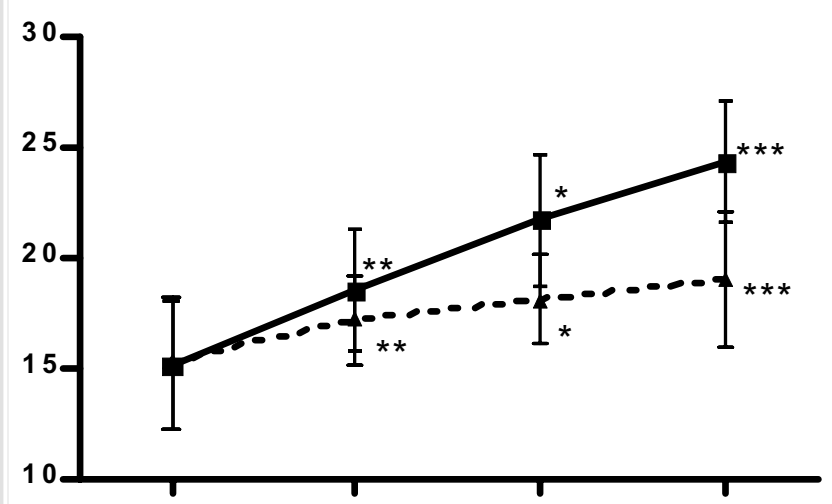

Rosca tríceps (Kg)

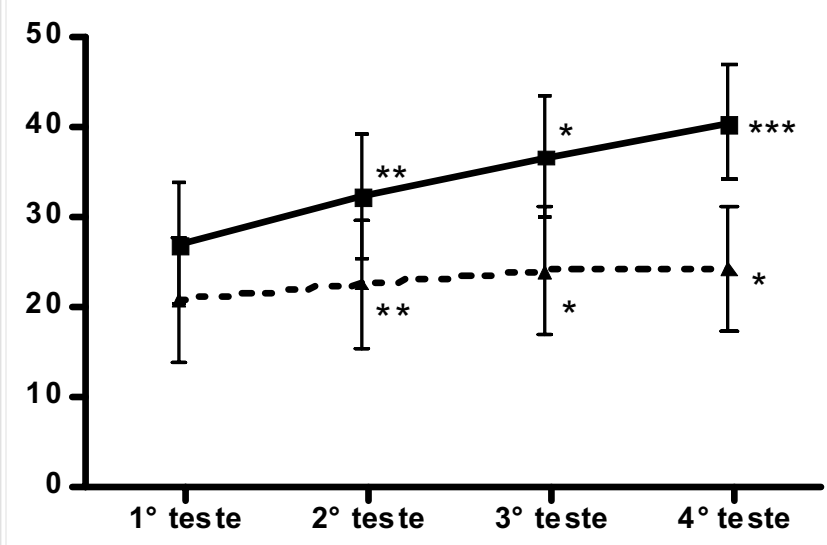

Figura 2. Análise intra-grupos com descrição por média e desviopadrão para os exercícios puxada pela frente $(A)$, rosca bíceps (B) e rosca tríceps (C). Linha cheia - G1; Linha tracejada - G2.

* diferença significativa em relação ao $1^{\circ}$ e $2^{\circ}$ teste $(p<0,05)$.

** diferença significativa em relação ao $1^{\circ}$ teste $(p<0,05)$.

*** diferença significativa em relação ao $1^{\circ}, 2^{\circ}$ e $3^{\circ}$ teste $(p<0,05)$. 
Tabela I: As análises inter-grupos foram expressas como média e desvio-padrão para todos exercícios do protocolo de treinamento.

\begin{tabular}{|c|c|c|c|c|c|c|c|c|}
\hline \multirow{3}{*}{$\begin{array}{c}\text { Leg press } 45^{\circ} \\
(\mathrm{Kg})\end{array}$} & \multirow[b]{2}{*}{ Gl } & \multicolumn{2}{|c|}{$1^{\circ}$ Teste } & \multicolumn{3}{|c|}{$2^{\circ}$ Teste } & $3^{\circ}$ Teste & $4^{\circ}$ Teste \\
\hline & & 90 & \pm 31.6 & 112,5 & \pm & 32 & $126,9 \pm 32 *$ & $138,1 \pm 30,5^{*}$ \\
\hline & $\mathrm{C} 2$ & $95,6 \pm$ & $\pm 15,2$ & 113,9 & \pm & 21,3 & $106,5 \pm 22$ & $107,6 \pm 22$ \\
\hline Extensão joelhos & Gl & $49,3 \pm$ & $\pm 16,5$ & 57,3 & \pm & 18,2 & $67,8 \pm 18,5$ & $64,5 \pm 18,5$ \\
\hline$(\mathrm{Kg})$ & $\mathrm{G}$ & $53,3 \pm$ & \pm 10 & 67,8 & \pm & 18.8 & $58,2 \pm 13$ & $59,5 \pm 13$ \\
\hline Flexão joelhos & Gl & $35,8 \pm$ & $\pm 12,9$ & 43,6 & \pm & 14,4 & $49,8 \pm 14,7$ & $54,5 \pm 14$ \\
\hline$(\mathrm{kg})$ & $\mathrm{C} 2$ & $36,8 \pm$ & \pm 3 & 39 & \pm & 15 & $34,8 \pm 16$ & $40,8 \pm 16$ \\
\hline Puxada $\mathrm{p} /$ frente & $\mathrm{Gl}$ & $32,5 \pm$ & 7 & 38,3 & \pm & 7,5 & $42,8 \pm 7,6$ & $47,1 \pm 7,8$ \\
\hline$(\mathrm{kg})$ & $\mathrm{C} 2$ & $37,5 \pm$ & 9 & 39,6 & \pm & 9 & $41,2 \pm 9,2$ & $42,1 \pm 9,1$ \\
\hline Rosca tríceps & Gl & $27,1 \pm$ & $\pm \quad 6,7$ & 32,3 & \pm & $6,9 *$ & $36,8 \pm 6,6^{*}$ & $40,6 \pm 6,3^{*}$ \\
\hline$(\mathrm{kg})$ & G2 & $20,9 \pm$ & $\pm \quad 7,1$ & 22,6 & \pm & 7,3 & $24,1 \pm 7$ & $24,4 \pm 7,4$ \\
\hline Rosca bíceps & Gl & $15,1 \pm$ & 2,9 & 18,5 & \pm & 2,7 & $21,7 \pm 3$ & $24,3 \pm 2,7^{*}$ \\
\hline$(\mathrm{kg})$ & $\mathrm{C} 2$ & $15,3 \pm$ & \pm 2 & 17,2 & \pm & 21 & $18,1 \pm 2$ & $19 \pm 3$ \\
\hline
\end{tabular}

*Diferença significativa em relação ao $G 1(p<0,05)$.

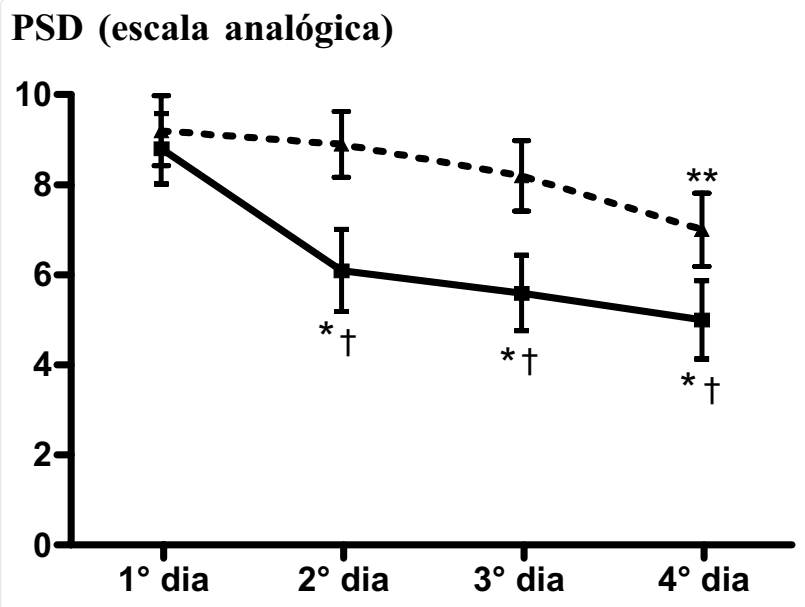

Figura 3: Análise intra e inter-grupos com descrição por média e desvio-padrão para percepção subjetiva de dor (PSD).

Linha cheia - G1; Linha tracejada - G2.

* Diferença significativa (intra-grupos) do $2^{\circ}, 3^{\circ}$ e $4^{\circ}$ dia em relação ao $1^{\circ}$ dia $(p<0,0001)$.

** Diferença significativa (intra-grupos) do $4^{\circ}$ dia em relação ao $1^{\circ}$ dia $(p<0,0001)$

† Diferença significativa (inter-grupos) do G1 em relação ao G2 $(p<0,001)$

\section{4- DISCUSSÃO}

Os parâmetros de aumento de força muscular, pela análise intergrupos, foram significativos para $\mathrm{G} 1$ em relação G2 nos exercícios leg press, rosca tríceps e rosca bíceps. Um dos fatores pertinentes ao retardo no processo de maturação da força muscular no G2 foi associado com os altos índices de dor muscular tardia associados à sobrecarga do treinamento inicial relatado pelos próprios voluntários. O estudo de Roth et al. ${ }^{16}$ corroborou com os nossos resultados, ou seja, eles verificaram queda da força muscular e índices significativos de dor muscular em jovens e idosos destreinados após nove semanas (três vezes por semana) de exercícios de força com alta intensidade. Para melhor fundamentação dos nossos resultados, o estudo de Coutts et al. ${ }^{17}$ que avaliaram jogadores de rugby durante seis semanas de treinamento com sobrecarga intensa mostraram redução significativa da força e potência muscular associado com dor muscular tardia e queda do balanço catabólico-anabólico.

Os nossos resultados relacionados a PSD mostraram-se bastante relevante no período de $48 \mathrm{~h}$ póssessão de treinamento. Em relação a literatura científica e a metodologia do nosso estudo não foram encontrados dados que conflitem ou corroborem nos resultados. Contudo, é relatado em alguns estudos que o período da dor muscular se intensificada em $48 \mathrm{~h}$, possivelmente pelo aumento da proliferação de neutrófilos imediatamente pós-sessão de treinamento ${ }^{18,19,20}$. No período entre 7-12h após a instalação do dano muscular ocorre uma migração de monócitos para região danificada ${ }^{18,19}$. Estes monócitos serão convertidos em macrófagos no músculo lesionado que ocasionam a sintetização das prostaglandinas ${ }^{18,20}$. Essa sintetização ativa os nociceptores periféricos que acionam fibras nervosas amielínicas do tipo III e IV, consequentemente, provoca a manifestação da dor ${ }^{21}$. 
Estes mecanismos fisiológicos hipoteticamente justificam o comportamento de dor intensa do G2 e da redução da força em relação a G1.

Outro mecanismo fisiológico relevante para sinalização dos receptores de dor, através dos nociceptores, é o influxo excessivo de íons de $\mathrm{Ca}^{2+}$ pela ruptura do retículo sarcoplasmático ou do sarcolema ${ }^{19}$. Assim, em relação a ruptura do retículo sarcoplasmático, este excesso de íons de $\mathrm{Ca}^{2+}$ proporciona a musculatura níveis de tetania, consequentemente, alto consumo energético e inibição da respiração celular ${ }^{19,22}$. Em relação a lesão no sarcolema, o excesso de influxo de íons de $\mathrm{Ca}^{2+}$ induz a força de cisalhamento que proporciona hipóxia localizada e débito energético, consequentemente, estimulam a produção de substâncias vasoativas ${ }^{22,23}$. É importante comentar que índices de dor muscular são prevalentes por danos nas linhas Z, túbulos T e sarcolema.

A principal hipótese para o aumento da força muscular do G1 em relação ao G2 foi a resposta adaptativa do sistema nervoso central em níveis de córtex motor $(\mathrm{CM})$, conseqüentemente, pela representação interna para uma melhor eficácia sináptica de neurônios em áreas corticais e sub-corticais ${ }^{14,24,25}$. Desta forma, o CM organiza o comando do movimento e envia este comando para um nível do centro inferior, numa seqüência relativamente bem definida: $\mathrm{CM}$, tálamo, gânglios da base, cerebelo, tronco cerebral, medula espinhal e músculo ${ }^{24,25}$. Assim, o procedimento adaptativo relacionado com as respostas do $\mathrm{CM}$ em relação aos exercícios de força estimula diretamente o aumento da capacidade de recrutamento e ativação das unidades motoras ${ }^{2,24,26}$.

As adaptações relacionadas as respostas neurais são proporcionais às alterações do número de sinapses corticais (angiogenêse cortical), força sináptica, topografia da estimulação evocada na representação dos movimentos, aumento da excitabilidade do motoneurônio á e redução da inibição pré-sináptica ${ }^{26,27}$. A proporcionalidade destas adaptações promove o melhor desenvolvimento da plasticidade neural ${ }^{25,27}$. Estes fatores foram proporcionalmente verificados no estudo de Jensen et al. ${ }^{24}$ que analisaram os níveis de disparo neural e os níveis de força em 24 indivíduos jovens e destreinados. Os indivíduos executaram exercícios de flexão de cotovelo (rosca bíceps) por quatro semanas e apresentaram ganhos de força de $31 \%$ associado ao aumento da excitabilidade cortical. Este resultado do estudo Jensen et al. ${ }^{24}$ corrobora com nosso estudo em relação à força muscular no exercício de rosca bíceps que apresentou ganhos de $60,9 \%$ ao final da $8^{\text {a }}$ semana de treinamento. Este fato pode estar relacionado ao tempo de treinamento (semanas), ou seja, Jensen et al. ${ }^{24}$ utilizou quatro semanas e no presente estudo foi utilizado oito semanas de treinamento fato que possivelmente proporcionou o dobro de valor encontrado por Jensen et al. ${ }^{24}$. No estudo de Hostler et al. ${ }^{28}$ foi verificado que o aumento progressivo de carga (principio da sobrecarga) com o exercício de rosca tríceps (extensão de cotovelo) mostrou-se efetivo para o aumento significativo de força muscular a partir do teste de 1RM durante o treinamento de força de oito semanas.

A resposta da força muscular para membros inferiores em relação às semanas iniciais de treinamento foi verificada por Ferri et al. ${ }^{29}$. Neste estudo os resultados mostraram ganhos significativos de força muscular, pelo teste de $1 \mathrm{RM}$, de $30 \%$ em idosos após 16 semanas de treinamento com exercícios de leg press. Em nossos resultados os ganhos de força no exercício de leg press foram de $27,8 \%$, assim os valores percentuais apresentaram total relevância entre o presente estudo e o estudo supracitado, mesmo que a diferença inter-semanas de treinamento tenha sido de $50 \%$ entre os estudos ${ }^{29}$. É possível comentar que a força muscular no estudo de Ferri et al. ${ }^{29}$ não apresentou aumento significativo em relação ao nosso estudo pelo fato de a partir da $8^{\mathrm{a}}$ semana de treinamento a atividade neural apresenta redução significativa das atividades de potencial evocado em relação ao início do treinamento, conseqüentemente, aumentando a prevalência dos fatores hipertróficos ${ }^{30,31}$. Assim o aumento da força muscular em relação aos fatores hipertróficos apresenta-se mais retardado pela dependência do aumento da área de secção transversa.

É importante comentar que o comportamento da força muscular relacionada as musculaturas do bíceps e tríceps em relação ao quadríceps varia pelo recrutamento aumentado de 47 unidades motoras durante a execução dos exercícios de extensão e flexão de cotovelo, desta forma, mantém um padrão estável de aceleração e desaceleração da execução do movimento $^{32}$. Este padrão de estabilidade é proporcional às adaptações prematuras em níveis de $\mathrm{CM}$ quando comparadas com os exercícios que envolvem grandes grupos musculares ${ }^{32,33}$. Estas adaptações são relativas ao aprimoramento das capacidades coordenativas dos sistemas musculares que acontece pelo direcionamento aumentado da inervação nas musculaturas ${ }^{14,33}$. Desta forma, o tempo de treinamento (semanas) influência diretamente nos ganhos de força através dos níveis coordenativos inter e intramuscular. 
A coordenação intermuscular apresenta a maior atividade do potencial de evolução motor em indivíduos destreinados ou sedentários durante o processo de adaptação neural a partir da $2^{\mathrm{a}}$ semana de treinamento ${ }^{14}$. Esta afirmação nos levou a optar pelo $1^{\circ}$ teste de $1 \mathrm{RM}$ da coleta de dados ocorrer ao final da $2^{\mathrm{a}}$ semana de treinamento, ou seja, por proporcionar recrutamento máximo das musculaturas estimuladas e seus sinergistas, conseqüentemente, a inibição das musculaturas antagonistas e finalmente mantendo a integridade das articulações através das musculaturas estabilizadoras $^{2,14,33}$. No G1 foi observado, em todos os exercícios, um padrão significativo de progressão da força muscular, porém, a partir da $4^{\mathrm{a}}$ semana de treinamento esta evolução mostrou-se bem relevante. Este fato pode estar diretamente associado pela melhora do recrutamento das unidades motoras de forma sincrônica a partir da evolução da coordenação intramuscular ${ }^{14,33}$.

Como fator de limitação do estudo seria necessário para melhor evolução dos resultados a utilização de um equipamento de eletromiografia para uma analise da progressão do potencial de ação evocado du- rante as semanas de treinamento. Outro fator relevante para completar o estudo seria uma análise bioquímica dos glóbulos brancos como marcador de índice inflamatório.

\section{5- CONCLUSÃO}

Podemos concluir que a sobrecarga progressiva imposta aos grupos musculares revelou aumento significativo da força muscular, do G1 em relação ao G2, ao longo das oito semanas de treinamento. Além disso, foi verificado que o princípio da sobrecarga de treinamento de forma controlada (aumento progressivo da sobrecarga em relação ao período de treinamento) apresentou redução significativa da dor muscular de acordo com as semanas de treinamento, quando relacionada à escala analógica de PSD G1 em relação ao G2. É importante comentar que mais estudos devem ser direcionados a essa linha de pesquisa, talvez com uma abordagem direcionada em níveis de potencial de ação neuromuscular de acordo com as semanas de treinamento.

Maior AS, Menuci T, Soares V, Souza LR, Gribov M, Simão R. Variation of the training overload in the behavior of the muscular force and subjective perception of pain in sedentary female. Medicina (Ribeirão Preto) 2008; 41 (2): 168-76.

ABSTRACT: The aim of the study was to analyze and compare gains of strength muscle and to observe the behavior of the subjective perception of pain (SPP) in the period eight weeks in sedentary women through two different programs from resistive exercises. The study was composed of 16 women $(27.5 \pm 4.8$ years; $57 \pm 3.9 \mathrm{~kg} ; 162.8 \pm 3.3 \mathrm{~cm} ; 21.4 \pm 1 \mathrm{~kg} / \mathrm{m} 2)$ healthy and sedentary. They were separate in two different groups: group 1 (G1) - during two initial weeks accomplished two sets of 10 repetitions with load that varied of 40 to $60 \%$ of the mass weight. However, 3th and 4th weeks they executed three sets of 10 repetitions to $80 \%$ of 1 RM. Among 5 th and 8th weeks it happened progressive increase of the set number for four maintaining the number of repetitions (10 repetitions) and same overload value; group 2 (G2) - program continuous in the exercise training of three sets of 10 repetitions was accomplished in the first two weeks with a load that varied of 40 to $60 \%$ of the corporal weight. Subsequent weeks the training was altered for four sets of 10 repetitions with an equivalent load to $80 \%$ of $1 \mathrm{RM}$ along the eight weeks of training. For analysis of the strength muscle the test of $1 \mathrm{RM}$ was used in the end of 2nd, 4th, 6th and 8th weeks with intention of to verify the behavior of the strength muscle starting from the displacement of loads and to optimize the training load. The exercises used in the training protocol and in the test of $1 \mathrm{RM}$ they were: leg press $45^{\circ}$, knee extension, knee flexion, lat pull down, elbow flexion, elbow extension. SPP was presented to each volunteer 48:00h after accomplishment of the test of 1RM. ANOVA Two Way following by the test post hoc of Bonferroni verified that the results intra-groups for G1 presented significant difference for all of the exercises in all the tests. However, G2 the significant difference was observed, in a large part of the exercises, only of the $3^{\circ}$ and $4^{\circ}$ test in relation to the $1^{\circ}$ test. For the analyses inter-groups the test Mann-Whitney for independent samples verified increase significant of strength muscle in the $\mathrm{G} 1$ in relation to $\mathrm{G} 2$ with exercises leg press $45^{\circ}$, elbow flexion, elbow extension. In relation to SPP a significant fall was observed in the analogical scale of $\mathrm{G} 1$ in relation to $\mathrm{G} 2(\mathrm{p}<0.001)$. The conclusion revealed that the progressive overload imposed G1 promoted increased gains of muscle strength than G2.

Key-Words: Muscle Strength. Physical Exercise. Muscle Pain. 


\section{REFERÊNCIA}

1 - American College of Sports Medicine - ACSM. Progression Models in Resistance Training for Healthy Adults. Med sci sports exerc 2002; 34(2): 364-80.

2 - Maior AS, Alves A. A contribuição dos fatores neurais em fases iniciais do treinamento de força muscular: uma revisão bibliográfica. Rev motriz 2003; 9(3): 161-8.

3 - Maior AS, Varallo AT, Matoso AGPS, Edmundo DA, De Oliveira MM, Minari VA. Resposta da força muscular em homens com a utilização de duas metodologias para o teste de 1RM. Rev bras cineantrop desempenho hum 2007; 9(2): 177-82.

4 - Maior AS, Alves Jr. CL, Ferraz FM, Menezes M, Carvalheira S, Simão R. Efeito hipotensivo dos exercícios resistidos em diferentes intervalos de recuperação. Rev Socerj 2007; 20(1): 53-9.

5 - Aoki MS, Pontes FL, Navarro F, Uchida MC, Bacurau FPB. Suplementação de carboidratos não reverte não reverte o efeito deletério do exercício de endurance sobre o subseqüente desempenho da força. Rev bras med esporte 2003; 9(5): 282-7

6 - Abadie BR, Wentworth MC. Prediction of one repetition maximal strength from a 5-10 repetition submaximal strength test in college-aged females. J exerc physiol online. 2000; 4(2): 1-6.

7 - Rhea MR, Alvar BA, Burkett LN, Ball SD. A meta-analysis to determine the dose response for strength development. Med sci sports exerc. 2003; 35(3): 456-64.

8 - Kemmler WK, Lauber D, Engelke K, Weineck J. Effects of single vs. multiple set resistance training on maximum strength and body composition in trained postmenopausal women. J strength cond res 2004; 18(4): 689-94.

9 - Moritani T, De Vries HA. Neural factors versus hypertrophy in the time course of muscle strength gain. Am j phys med1979; 58: 115-30.

10 - Gruber M, Taube W, Gollhofer A, Beck S, Amtage F, Schubert $\mathrm{M}$. Training-specific adaptations of $\mathrm{H}$ - and stretch reflexes in human soleus muscle. J mot behav 2007; 39(1): 68-78.

11 - Van Cutsem M, Duchateau J, Hainaut K. Changes in single motor unit behaviour contribute to the increase in contraction speed after dynamic training in humans. J physiol (London) 1998; 15(51): 295-305.

12 - Gruber M, Gruber SB, Taube W, Schubert M, Beck SC, Gollhofer A. Differential effects of ballistic versus sensorimotor training on rate of force development and neural activation in humans. J strength cond res 2007; 21(1): 274-82.

13- Loveless DJ, Weber CL, Haseler LJ, Schneider DA. Maximal leg-strength training improves cycling economy in previously untrained men. Med sci sports exerc 2005; 37(7): 1231-6.

14 - Griffin L, Cafarelli E. Resistance training: cortical, spinal, and motor unit adaptation. Can j appl physiol 2005; 30(3): 328-40.

15 - Cook DB, Patrick J, O'Connor O, Ray C. Muscle pain perception and sympathetic nerve activity to exercise during opioid modulation. Am j physiol regul integr comp physiol 2000; 279(5):1565-73.

16 - Roth SM, Martel GF, Ivey FM, Lemmer JT, Metter EJ, Hurley BF, Rogers MA. High-volume, heavy-resistance strength training and muscle damage in young and older women. $J$ appl physiol 2000; 88(3): 1112-8.
17 - Coutts A, Reaburn P, Piva TJ, Murphy A. Changes in selected biochemical, muscular strength, power, and endurance measures during deliberate overreaching and tapering in rugby league players. Int j sports med 2007; 28(2): 116-24.

18 - Butterfield TA, Best TM, Merrick MA. The dual roles of neutrophils and macrophages in inflammation: a critical balance between tissue damage and repair. J Athl Train 2006; 41(4): 457-65.

19 - Armstrong RB, Warren GL, Warren JA. Mechanics of exercise-induced muscle fiber injury. Sports med 1991; 12: 184-207.

20 - Tidball JG. Interactions between muscle and the immune system during modified musculoskeletal loading. Clin orthop relat res 2002; (403 Suppl): S100-9.

21 - Teixeira ML, Lin TY, Kaziyama HHS, Ramos CA. Fisiopatologia da dor músculo-esquelética. Rev med (São Paulo) 2001; 80 (ed. especial 1): 63-77.

22 - Liu J, Yeo HC, Overvik-Douki E, Hagen T, Doniger SJ, Chyu DW, Brooks GA, Ames BN. Chronically and acutely exercised rats: biomarkers of oxidative stress and endogenous antioxidants. J appl physiol 2000; 89(1): 21-8.

23 - Stark G. Functional consequences of oxidative membrane damage. J membr biol 2005; 205(1): 1-16.

24 - Jensen JL, Marstrand PC, and Nielsen JB. Motor skill training and strength training are associated with different plastic changes in the central nervous system. J appl physiol 2005; 99: 1558-68.

25 - Adkins DL, Boychuk J, Remple MS, Kleim JA. Motor training induces experience-specific patterns of plasticity across motor cortex and spinal cord. J appl physiol 2006; 101(6): 1776-82.

26 - Monfils MH, Plautz EJ, and Kleim JA. In search of the motor engram: motor map plasticity as a mechanism for encoding motor experience. Neuroscientist 2005; 11: 471-83.

27 - Lagerquist O, Zehr EP, Docherty D. Increased spinal reflex excitability is not associated with neural plasticity underlying the cross-education effect. J appl physiol 2006; 100(1): 8390 .

28 - Hostler D, Crill MT, Hagerman FC, Staron RS. The effectiveness of $0.5-\mathrm{lb}$ increments in progressive resistance exercise. J strength cond res 2001; 15(1): 86-91.

29 - Ferri A, Scaglioni G, Pousson M, Capodaglio P, Van Hoecke $\mathrm{J}$, Narici MV. Strength and power changes of the human plantar flexors and knee extensors in response to resistance training in old age. Acta physiol scand 2003; 177(1): 69-78.

30 - Enoka RM. Neural adaptations with chronic Physical activity. J biomech 1997; 30(5): 447-55.

31 - Carolan B, Cafarelli E. Adaptations in coactivation after isometric resistance training. J appl physiol 1992; 73: 911-7.

32 - Garland SJ, Cooke JD, Miller KJ, Ohtsuki T, Ivanova T. Motor unit activity during human single joint movements. J neurophysiol 1996; 76(3): 1982-90.

33 - Blazevich AJ, Gill ND, Zhou S. Intra- and intermuscular variation in human quadriceps femoris architecture assessed in vivo. J anat 2006; 209(3): 289-310.

Recebido para publicação em 26/09/2007.

Aprovado para publicação em 27/03/2008 